\title{
A new theorem on the existence of the Riemann-Stieltjes integral and an improved version of the Loéve-Young inequality
}

\section{Rafał M Łochowski*}

"Correspondence:

rlocho314@gmail.com

Department of Mathematics and

Mathematical Economics, Warsaw

School of Economics,

Madalińskiego 6/8, Warsaw, 02-513,

Poland

\begin{abstract}
Using the notion of the truncated variation we obtain a new theorem on the existence and estimation of the Riemann-Stieltjes integral. As a special case of this theorem we obtain an improved version of the Loéve-Young inequality for the Riemann-Stieltjes integrals driven by irregular signals. Using this result we strengthen some results of Lyons on the existence of solutions of integral equations driven by moderately irregular signals.
\end{abstract}

MSC: Primary 26A42; secondary 45G10

Keywords: the Loéve-Young inequality; the Riemann-Stieltjes integral; irregular signals

\section{Introduction}

The purpose of this paper is to investigate the top-down structure of the Riemann-Stieltjes integral and to state some general condition guaranteeing the existence of this integral, expressed in terms of the functional called truncated variation.

The simplest (and rather not surprising) case where the Riemann-Stieltjes integral $\int_{a}^{b} f \mathrm{~d} g$ (RSI in short) exists, is the situation when the integrand and the integrator have no common points of discontinuity, the former is bounded and the latter has finite total variation. We will prove a general theorem (Theorem 1) encompassing this situation as well as the more interesting case when the RSI exists, namely when the integrand and the integrator have a possibly unbounded variation, but they have finite $p$-variation and $q$-variation, respectively, with $p>1, q>1$ and $p^{-1}+q^{-1}>1$. The latter result is due to Young [1], p.264, theorem on Stieltjes integrability. For $f:[a ; b] \rightarrow \mathbb{R}$ and $p>0$, the $p$-variation, which we will denote by $V^{p}(f ;[a ; b])$, is defined as

$$
V^{p}(f,[a ; b])=\sup _{n} \sup _{a \leq t_{1}<t_{2}<\cdots<t_{n} \leq b} \sum_{i=1}^{n-1}\left|f\left(t_{i}\right)-f\left(t_{i-1}\right)\right|^{p}
$$

The aforementioned Theorem 1 provides also an upper bound for the difference

$$
\left|\int_{a}^{b} f \mathrm{~d} g-f(a)[g(b)-g(a)]\right| .
$$

(c) 2015 Łochowski. This article is distributed under the terms of the Creative Commons Attribution 4.0 International License (http://creativecommons.org/licenses/by/4.0/), which permits unrestricted use, distribution, and reproduction in any medium, provided you give appropriate credit to the original author(s) and the source, provide a link to the Creative Commons license, and indicate if changes were made. 
As a special case of this bound we will obtain the following, improved version of the classical Loéve-Young inequality:

$$
\begin{aligned}
& \left|\int_{a}^{b} f \mathrm{~d} g-f(a)[g(b)-g(a)]\right| \\
& \leq C_{p, q}\left(V^{p}(f,[a ; b])\right)^{1-1 / q}\|f\|_{\text {osc },[a ; b]}^{1+p / q-p}\left(V^{q}(g,[a ; b])\right)^{1 / q},
\end{aligned}
$$

where $C_{p, q}$ is some constant depending on $p$ and $q$ only and $\|f\|_{\text {osc, },[a ; b]}:=\sup _{a \leq s<t \leq b} \mid f(t)-$ $f(s) \mid$. The original Loéve-Young estimate, published in 1936 in [1], reads

$$
\left|\int_{a}^{b} f \mathrm{~d} g-f(a)[g(b)-g(a)]\right| \leq \zeta\left(p^{-1}+q^{-1}\right)\left(V^{p}(f,[a ; b])\right)^{1 / p}\left(V^{q}(g,[a ; b])\right)^{1 / q}
$$

where $\zeta(r)=\sum_{k=1}^{+\infty} k^{-r}$ is the famous Riemann zeta function. We say that our bound is improved version of the Loéve-Young inequality since the value of the constant $C_{p, q}$ (although possibly greater than $\left.\zeta\left(p^{-1}+q^{-1}\right)\right)$ is irrelevant in applications while the fact that the term $\left(V^{p}(f,[a ; b])\right)^{1 / p}$ in the original Loéve-Young estimate is replaced in our estimate by $\left(V^{p}(f,[a ; b])\right)^{1-1 / q}\|f\|_{\text {osc, }[a ; b]}^{1+p / q-p}$ (notice that $1 / p>1-1 / q$ and always $\left(V^{p}(f,[a ; b])\right)^{1 / p-(1-1 / q)} \geq$ $\left.\|f\|_{\text {osc },[a ; b]}^{1+p / q-p}\right)$ makes it possible to obtain stronger results. For example, Proposition $1 \mathrm{ob}-$ tained in Section 3 with the help of (1) is a genuine improvement upon earlier known results of this type.

Let us comment shortly how the results on the existence of the RSI were obtained so far. The original Young's proof utlilised elementary but clever induction argument for finite sequences. Another proof of the Young theorem may be found in [2], Chapter 6, where integral estimates based on control function and the Loéve-Young inequality are used. This approach is further applied in the rough-path theory setting. Further generalisations of Young's theorem are possible, with $p$-variation replaced by the more general $\varphi$-variation:

$$
V^{\varphi}(f,[a ; b])=\sup _{n} \sup _{a \leq t_{1}<t_{2}<\cdots<t_{n} \leq b} \sum_{i=1}^{n-1} \varphi\left(\left|f\left(t_{i}\right)-f\left(t_{i-1}\right)\right|\right),
$$

where $\varphi:[0 ;+\infty) \rightarrow[0 ;+\infty)$ is a Young function, i.e. a convex, strictly increasing function starting from 0 (see for example [3, 4] and for a survey of other results of this type, see [5], Chapter 3, [6], Section 4.4).

However, as far as we know, Theorem 1 is a new result on the existence of the RSI. The proof of Theorem 1 utilises simple properties of the truncated variation and multiple application of the summation by parts. Similarly, no version of the Loéve-Young inequality as estimate (1), as far as we know, has appeared so far (see the detailed historical notes on the Loéve-Young inequality in [5], pp.212-214). We conjecture that using Theorem 1 one may also obtain a variation of the Loéve-Young inequality for $\varphi$-variation (see [5], Theorem 3.89, Corollary 3.90, or [6], Theorem 4.40). We intend to deal with this conjecture in the future.

After having obtained these results we were able, following Lyons [7], and Lyons et al. [8], to solve few types of integral equations driven by moderately irregular signals. By moderately irregular signals we mean continuous signals with finite $p$-variation, where $p \in(1 ; 2)$. It is well known that for higher degrees of irregularity, corresponding to $p \geq 2$, one needs to 
construct approximations of integral equations, to consider terms of new type (like Lévy's area). We believe that the truncated variation approach for such paths is also possible and this will be a topic of our further research.

Let us comment shortly on the organisation of the paper. In the next section we prove a general theorem on the existence of the Riemann-Stieltjes integral, expressed in terms of the truncated variation functionals and derive from it the stronger version of the LoéveYoung inequality. Next, in Section 3, we deal with the applications of this result to few types of integral equations driven by moderately irregular signals.

\section{A theorem on the existence of the Riemann-Stieltjes integral}

In this section we will prove a general theorem on the existence of the RSI $\int_{a}^{b} f \mathrm{~d} g$ formulated in terms of the truncated variation. We will assume that both - integrand $f:[a ; b] \rightarrow$ $\mathbb{R}$ and integrator $g:[a ; b] \rightarrow \mathbb{R}$ are regulated functions. Let us state the definition of the truncated variation and recall the definition of a regulated function.

For $f:[a ; b] \rightarrow \mathbb{R}$ its truncated variation with the truncation parameter $\delta \geq 0$ will be denoted by $\operatorname{TV}^{\delta}(f,[a ; b])$. It may be simply defined as the greatest lower bound for the total variation of any function $g:[a ; b] \rightarrow \mathbb{R}$, uniformly approximating $f$ with the accuracy $\delta / 2$,

$$
\operatorname{TV}^{\delta}(f,[a ; b]):=\inf \left\{\operatorname{TV}(g,[a ; b]):\|f-g\|_{\infty,[a ; b]} \leq \delta / 2\right\}
$$

$\|f-g\|_{\infty,[a ; b]}$ denotes here $\sup _{a \leq t \leq b}|f(t)-g(t)|$ and the total variation $\operatorname{TV}(g,[a ; b])$ is defined as

$$
\operatorname{TV}(g,[a ; b]):=\sup _{n} \sup _{a \leq t_{1}<t_{2}<\cdots<t_{n} \leq b} \sum_{i=1}^{n-1}\left|g\left(t_{i}\right)-g\left(t_{i-1}\right)\right| .
$$

It appears that the truncated variation $\operatorname{TV}^{\delta}(f,[a ; b])$ is finite for any $\delta>0$ iff $f$ is regulated (cf. [9], Fact 2.2) and then for any $\delta>0$ the following equality holds:

$$
\operatorname{TV}^{\delta}(f,[a ; b])=\sup _{n} \sup _{a \leq t_{1}<t_{2}<\cdots<t_{n} \leq b} \sum_{i=1}^{n-1} \max \left\{\left|f\left(t_{i}\right)-f\left(t_{i-1}\right)\right|-\delta, 0\right\}
$$

(cf. [10], Theorem 4).

Let us recall that a function $h:[a ; b] \rightarrow \mathbb{R}$ is regulated if there exist one-sided finite limits $\lim _{t \rightarrow a_{+}} h(t)$ and $\lim _{t \rightarrow b_{-}} h(t)$, and for any $t \in(a ; b)$ there exist one-sided finite limits $\lim _{t \rightarrow x-} h(t)$ and $\lim _{t \rightarrow x+} h(t)$.

We will also need the following result (cf. [10], Theorem 4): for any regulated function $f:[a ; b] \rightarrow \mathbb{R}$ and $\delta>0$ there exists a regulated function $f^{\delta}:[a ; b] \rightarrow \mathbb{R}$ such that $\| f-$ $f^{\delta} \|_{\infty,[a ; b]} \leq \delta / 2$ and

$$
\operatorname{TV}^{0}\left(f^{\delta},[a ; b]\right)=\operatorname{TV}^{\delta}(f,[a ; b])
$$

From equation (2), it directly follows that the truncated variation is a superadditive functional of the interval, i.e. for any $d \in(a ; b)$

$$
\operatorname{TV}^{\delta}(f,[a ; b]) \geq \operatorname{TV}^{\delta}(f,[a ; d])+\operatorname{TV}^{\delta}(f,[d ; b])
$$


Moreover, we also have the following easy estimate of the truncated variation of a function $f$ perturbed by some other function $h$ :

$$
\operatorname{TV}^{\delta}(f+h,[a ; b]) \leq \operatorname{TV}^{\delta}(f,[a ; b])+\operatorname{TV}^{0}(h,[a ; b]),
$$

which stems directly from the inequality, for $a \leq s<t \leq b$,

$$
\max \{|f(t)+h(t)-\{f(s)+h(s)\}|-\delta, 0\} \leq \max \{|f(t)-f(s)|-\delta, 0\}+|h(t)-h(s)| .
$$

Theorem 1 Let $f, g:[a ; b] \rightarrow \mathbb{R}$ be two regulated functions which have no common points of discontinuity. Let $\eta_{0} \geq \eta_{1} \geq \cdots$ and $\theta_{0} \geq \theta_{1} \geq \cdots$ be two sequences of non-negative numbers, such that $\eta_{k} \downarrow 0, \theta_{k} \downarrow 0$ as $k \rightarrow+\infty$. Define $\eta_{-1}:=\sup _{a \leq t \leq b}|f(t)-f(a)|$ and

$$
S:=\sum_{k=0}^{+\infty} 2^{k} \eta_{k-1} \cdot \operatorname{TV}^{\theta_{k}}(g,[a ; b])+\sum_{k=0}^{\infty} 2^{k} \theta_{k} \cdot \operatorname{TV}^{\eta_{k}}(f,[a ; b])
$$

If $S<+\infty$ then the Riemann-Stieltjes integral $\int_{a}^{b} f \mathrm{~d} g$ exists and one has the following estimate :

$$
\left|\int_{a}^{b} f \mathrm{~d} g-f(a)[g(b)-g(a)]\right| \leq S .
$$

Remark 1 The assumption that $f$ and $g$ have no common points of discontinuity is necessary for the existence of the RSI $\int_{a}^{b} f \mathrm{~d} g$. When more general integrals are considered (e.g. the Moore-Pollard integral, $c f$. [1], p.263), we may weaken this assumption and assume that $f$ and $g$ have no common one-sided discontinuities.

The proof of Theorem 1 will be based on the following lemma.

Lemma 1 Let $f, g:[a ; b] \rightarrow \mathbb{R}$ be two regulated functions. Let $c=t_{0}<t_{1}<\cdots<t_{n}=d$ be any partition of the interval $[c ; d] \subset[a ; b]$ and let $\xi_{0}=c$ and $\xi_{1}, \ldots, \xi_{n}$ be such that $t_{i-1} \leq \xi_{i} \leq t_{i}$ for $i=1,2, \ldots, n$. Then for $\delta_{-1}:=\sup _{c \leq t \leq d}|f(t)-f(c)|, \delta_{0} \geq \delta_{1} \geq \cdots \geq \delta_{r} \geq 0$ and $\varepsilon_{0} \geq \varepsilon_{1} \geq$ $\cdots \geq \varepsilon_{r} \geq 0$ the following estimate holds:

$$
\begin{aligned}
& \left|\sum_{i=1}^{n} f\left(\xi_{i}\right)\left[g\left(t_{i}\right)-g\left(t_{i-1}\right)\right]-f(c)[g(d)-g(c)]\right| \\
& \quad \leq \sum_{k=0}^{r} 2^{k} \delta_{k-1} \cdot \mathrm{TV}^{\varepsilon k}(g,[c ; d])+\sum_{k=0}^{r} 2^{k} \varepsilon_{k} \cdot \mathrm{TV}^{\delta_{k}}(f,[c ; d])+n \delta_{r} \varepsilon_{r} .
\end{aligned}
$$

Proof Denote $\varepsilon=\varepsilon_{0}$, by summation by parts, we have the following equality:

$$
\begin{gathered}
\sum_{i=1}^{n} f\left(\xi_{i}\right)\left[g\left(t_{i}\right)-g\left(t_{i-1}\right)\right]-f(c)[g(d)-g(c)] \\
=\sum_{i=1}^{n}\left[f\left(\xi_{i}\right)-f(c)\right]\left[g^{\varepsilon}\left(t_{i}\right)-g^{\varepsilon}\left(t_{i-1}\right)\right]
\end{gathered}
$$




$$
\begin{aligned}
& +\sum_{i=1}^{n}\left[f\left(\xi_{i}\right)-f(c)\right]\left[g\left(t_{i}\right)-g^{\varepsilon}\left(t_{i}\right)-\left\{g\left(t_{i-1}\right)-g^{\varepsilon}\left(t_{i-1}\right)\right\}\right] \\
= & \sum_{i=1}^{n}\left[f\left(\xi_{i}\right)-f(c)\right]\left[g^{\varepsilon}\left(t_{i}\right)-g^{\varepsilon}\left(t_{i-1}\right)\right] \\
& +\sum_{i=1}^{n}\left[g(d)-g^{\varepsilon}(d)-\left\{g\left(t_{i-1}\right)-g^{\varepsilon}\left(t_{i-1}\right)\right\}\right]\left[f\left(\xi_{i}\right)-f\left(\xi_{i-1}\right)\right],
\end{aligned}
$$

where $g^{\varepsilon}:[c ; d] \rightarrow \mathbb{R}$ is regulated and such that

$$
\left\|g-g^{\varepsilon}\right\|_{\infty,[c ; d]} \leq \frac{1}{2} \varepsilon \quad \text { and } \quad \operatorname{TV}^{0}\left(g^{\varepsilon},[c ; d]\right)=\operatorname{TV}^{\varepsilon}(g,[c ; d])
$$

Similarly, for $\delta=\delta_{0}$ we may write

$$
\begin{aligned}
& \sum_{i=1}^{n} {\left[g(d)-g^{\varepsilon}(d)-\left\{g\left(t_{i-1}\right)-g^{\varepsilon}\left(t_{i-1}\right)\right\}\right]\left[f\left(\xi_{i}\right)-f\left(\xi_{i-1}\right)\right] } \\
&= \sum_{i=1}^{n}\left[g(d)-g^{\varepsilon}(d)-\left\{g\left(t_{i-1}\right)-g^{\varepsilon}\left(t_{i-1}\right)\right\}\right]\left[f^{\delta}\left(\xi_{i}\right)-f^{\delta}\left(\xi_{i-1}\right)\right] \\
& \quad+\sum_{i=1}^{n}\left[f\left(\xi_{i}\right)-f^{\delta}\left(\xi_{i}\right)-\left\{f(c)-f^{\delta}(c)\right\}\right]\left[\left\{g\left(t_{i}\right)-g^{\varepsilon}\left(t_{i}\right)\right\}-\left\{g\left(t_{i-1}\right)-g^{\varepsilon}\left(t_{i-1}\right)\right\}\right],
\end{aligned}
$$

where $f^{\delta}:[c ; d] \rightarrow \mathbb{R}$ is regulated and such that

$$
\left\|f-f^{\delta}\right\|_{\infty,[c ; d]} \leq \frac{1}{2} \delta \quad \text { and } \quad \operatorname{TV}^{0}\left(f^{\delta},[c ; d]\right)=\operatorname{TV}^{\delta}(f,[c ; d])
$$

Since $\operatorname{TV}^{0}\left(g^{\varepsilon},[c ; d]\right)=\operatorname{TV}^{\varepsilon}(g,[c ; d]), \operatorname{TV}^{0}\left(f^{\delta},[c ; b]\right)=\operatorname{TV}^{\delta}(f,[c ; d]),\left\|g-g^{\varepsilon}\right\|_{\infty,[c ; d]} \leq \varepsilon / 2$ and $\left\|f-f^{\delta}\right\|_{\infty,[c ; d]} \leq \delta / 2$, from (5) and (6) we have the following estimate:

$$
\begin{aligned}
& \left|\sum_{i=1}^{n} f\left(\xi_{i}\right)\left[g\left(t_{i}\right)-g\left(t_{i-1}\right)\right]-f(c)[g(d)-g(c)]\right| \\
& \quad \leq \sup _{c \leq t \leq d}|f(t)-f(c)| \cdot \operatorname{TV}^{\varepsilon}(g,[c ; d])+\varepsilon \cdot \operatorname{TV}^{\delta}(f,[c ; d])+n \delta \varepsilon .
\end{aligned}
$$

Denote $g_{1}:=g-g^{\varepsilon}, f_{1}:=f-f^{\delta}$ on $[c ; d]$. By (5) and (6), instead of the last summand $n \delta \varepsilon$ in (7) we may write the estimate

$$
\begin{aligned}
& \left|\sum_{i=1}^{n}\left[f\left(\xi_{i}\right)-f^{\delta}\left(\xi_{i}\right)-\left\{f(c)-f^{\delta}(c)\right\}\right]\left[\left\{g\left(t_{i}\right)-g^{\varepsilon}\left(t_{i}\right)\right\}-\left\{g\left(t_{i-1}\right)-g^{\varepsilon}\left(t_{i-1}\right)\right\}\right]\right| \\
& \quad=\left|\sum_{i=1}^{n}\left[f_{1}\left(\xi_{i}\right)-f_{1}(c)\right]\left[g_{1}\left(t_{i}\right)-g_{1}\left(t_{i-1}\right)\right]\right| \\
& \quad \leq \sup _{c \leq t \leq d}\left|f_{1}(t)-f_{1}(c)\right| \cdot \mathrm{TV}^{\varepsilon_{1}}\left(g_{1},[c ; d]\right)+\varepsilon_{1} \cdot \mathrm{TV}^{\delta_{1}}\left(f_{1},[c ; d]\right)+n \delta_{1} \varepsilon_{1} \\
& \quad \leq \delta \cdot \mathrm{TV}^{\varepsilon_{1}}\left(g_{1},[c ; d]\right)+\varepsilon_{1} \cdot \mathrm{TV}^{\delta_{1}}\left(f_{1},[c ; d]\right)+n \delta_{1} \varepsilon_{1},
\end{aligned}
$$


where the last but one inequality in (8) follows by the same reasoning for $f_{1}$ and $g_{1}$ as inequality (5) for $f$ and $g$. Repeating these arguments, by induction we get

$$
\begin{aligned}
& \left|\sum_{i=1}^{n} f\left(\xi_{i}\right)\left[g\left(t_{i}\right)-g\left(t_{i-1}\right)\right]-f(c)[g(d)-g(c)]\right| \\
& \quad \leq \sum_{k=0}^{r} \delta_{k-1} \cdot \operatorname{TV}^{\varepsilon_{k}}\left(g_{k},[c ; d]\right)+\sum_{k=0}^{r} \varepsilon_{k} \cdot \operatorname{TV}^{\delta_{k}}\left(f_{k},[c ; d]\right)+n \delta_{r} \varepsilon_{r},
\end{aligned}
$$

where $\delta_{-1}:=\sup _{c \leq t \leq c}|f(t)-f(a)|, g_{0} \equiv g, f_{0} \equiv f$ and for $k=1,2, \ldots, r, g_{k}:=g_{k-1}-g_{k-1}^{\varepsilon_{k-1}}$, $f_{k}:=f_{k-1}-f_{k-1}^{\delta_{k-1}}$ are defined similarly as $g_{1}$ and $f_{1}$.

Since $\varepsilon_{k} \leq \varepsilon_{k-1}$ for $k=1,2, \ldots, r$, by (3) and the fact that the function $\delta \mapsto \operatorname{TV}^{\delta}(h,[c ; d])$ is non-increasing, we estimate

$$
\begin{aligned}
\operatorname{TV}^{\varepsilon_{k}}\left(g_{k},[c ; d]\right) & =\operatorname{TV}^{\varepsilon_{k}}\left(g_{k-1}-g_{k-1}^{\varepsilon_{k-1}},[c ; d]\right) \\
& \leq \operatorname{TV}^{\varepsilon_{k}}\left(g_{k-1},[c ; d]\right)+\operatorname{TV}^{0}\left(g_{k-1}^{\varepsilon_{k-1}},[c ; d]\right) \\
& =\operatorname{TV}^{\varepsilon_{k}}\left(g_{k-1},[c ; d]\right)+\operatorname{TV}^{\varepsilon_{k-1}}\left(g_{k-1},[c ; d]\right) \\
& \leq 2 \operatorname{TV}^{\varepsilon_{k}}\left(g_{k-1},[c ; d]\right) .
\end{aligned}
$$

Hence, by recursion, for $k=1,2, \ldots, r$,

$$
\operatorname{TV}^{\varepsilon_{k}}\left(g_{k},[c ; d]\right) \leq 2^{k} \mathrm{TV}^{\varepsilon_{k}}(g,[c ; d]) .
$$

Similarly, for $k=1,2, \ldots, r$, we have

$$
\operatorname{TV}^{\delta_{k}}\left(f_{k},[c ; d]\right) \leq 2^{k} \mathrm{TV}^{\delta_{k}}(f,[c ; d]) .
$$

By (9) and last two estimates we get the desired estimate.

Remark 2 Notice that starting in (5) from the summation by parts, then splitting the difference $f\left(\xi_{i}\right)-f\left(\xi_{i-1}\right)$ :

$$
\begin{aligned}
& \sum_{i=1}^{n} f\left(\xi_{i}\right)\left[g\left(t_{i}\right)-g\left(t_{i-1}\right)\right]-f(c)[g(d)-g(c)] \\
& =\sum_{i=1}^{n}\left[g(d)-g\left(t_{i-1}\right)\right]\left[f\left(\xi_{i}\right)-f\left(\xi_{i-1}\right)\right]=\sum_{i=1}^{n}\left[g(d)-g\left(t_{i-1}\right)\right]\left[f^{\delta}\left(\xi_{i}\right)-f^{\delta}\left(\xi_{i-1}\right)\right] \\
& \quad+\sum_{i=1}^{n}\left[g(d)-g\left(t_{i-1}\right)\right]\left[f\left(\xi_{i}\right)-f^{\delta}\left(\xi_{i}\right)-\left\{f\left(\xi_{i-1}\right)-f^{\delta}\left(\xi_{i-1}\right)\right\}\right]
\end{aligned}
$$

and proceeding similarly to the proof of Lemma 1 we get the symmetric estimate

$$
\begin{aligned}
& \left|\sum_{i=1}^{n} f\left(\xi_{i}\right)\left[g\left(t_{i}\right)-g\left(t_{i-1}\right)\right]-f(c)[g(d)-g(c)]\right| \\
& \quad \leq \sum_{k=0}^{r} 2^{k} \varepsilon_{k-1} \cdot \mathrm{TV}^{\delta_{k}}(f,[c ; d])+\sum_{k=0}^{r} 2^{k} \delta_{k} \cdot \mathrm{TV}^{\varepsilon_{k}}(g,[c ; d])+n \delta_{r} \varepsilon_{r},
\end{aligned}
$$

where $\varepsilon_{-1}=\sup _{c \leq t \leq d}|g(d)-g(t)|$. 
Remark 3 Setting in Lemma $1, n=1$ for any $\xi \in[c ; d]$ we get the estimate

$$
\begin{aligned}
& |(f(\xi)-f(c))[g(d)-g(c)]| \\
& \quad \leq \sum_{k=0}^{r} 2^{k} \delta_{k-1} \cdot \mathrm{TV}^{\varepsilon_{k}}(f,[c ; d])+\sum_{k=0}^{r} 2^{k} \varepsilon_{k} \cdot \mathrm{TV}^{\delta_{k}}(g,[c ; d])+n \delta_{r} \varepsilon_{r}
\end{aligned}
$$

and similarly, setting in Remark $2 n=1$ we get a similar estimate, where the right side of (11) is replaced by the right side of (10).

Now we proceed to the proof of Theorem 1.

Proof It is enough to prove that for any two partitions

$$
\begin{aligned}
& \pi=\left\{a=a_{0}<a_{1}<\cdots<a_{l}=b\right\}, \\
& \rho=\left\{a=b_{0}<b_{1}<\cdots<b_{m}=b\right\},
\end{aligned}
$$

where $v_{i} \in\left[a_{i-1} ; a_{i}\right], \xi_{j} \in\left[b_{j-1} ; b_{j}\right], i=1,2, \ldots, l, j=1,2, \ldots, m$, the difference

$$
\left|\sum_{i=1}^{l} f\left(v_{i}\right)\left[g\left(a_{i}\right)-g\left(a_{i-1}\right)\right]-\sum_{j=1}^{m} f\left(\xi_{j}\right)\left[g\left(b_{j}\right)-g\left(b_{j-1}\right)\right]\right|
$$

is as small as we please, provided that the meshes of the partitions $\pi$ and $\rho$, defined as

$$
\operatorname{mesh}(\pi):=\max _{i=1,2, \ldots, l}\left(a_{i}-a_{i-1}\right), \quad \operatorname{mesh}(\rho):=\max _{j=1,2, \ldots, m}\left(b_{j}-b_{j-1}\right)
$$

respectively, are sufficiently small.

Define

$$
\sigma=\pi \cup \rho=\left\{a=s_{0}<s_{1}<\cdots<s_{n}=b\right\}
$$

and for $i=1,2, \ldots, l$ consider

$$
\left|f\left(v_{i}\right)\left[g\left(a_{i}\right)-g\left(a_{i-1}\right)\right]-\sum_{k: s_{k-1}, s_{k} \in\left[a_{i-1} ; a_{i}\right]} f\left(s_{k-1}\right)\left[g\left(s_{k}\right)-g\left(s_{k-1}\right)\right]\right| .
$$

We estimate

$$
\begin{aligned}
\mid f\left(v_{i}\right) & {\left[g\left(a_{i}\right)-g\left(a_{i-1}\right)\right]-\sum_{k: s_{k-1}, s_{k} \in\left[a_{i-1} ; a_{i}\right]} f\left(s_{k-1}\right)\left[g\left(s_{k}\right)-g\left(s_{k-1}\right)\right] \mid } \\
\leq & \left|f\left(v_{i}\right)\left[g\left(a_{i}\right)-g\left(a_{i-1}\right)\right]-f\left(a_{i-1}\right)\left[g\left(a_{i}\right)-g\left(a_{i-1}\right)\right]\right| \\
& +\left|\sum_{k: s_{k-1}, s_{k} \in\left[a_{i-1} ; a_{i}\right]} f\left(s_{k-1}\right)\left[g\left(s_{k}\right)-g\left(s_{k-1}\right)\right]-f\left(a_{i-1}\right)\left[g\left(a_{i}\right)-g\left(a_{i-1}\right)\right]\right| .
\end{aligned}
$$

Recall the definition of $S$. If there exists $N=0,1,2, \ldots$ such that $\eta_{N}=0$ or $\theta_{N}=0$ then $\operatorname{TV}(f,[a ; b])$ or $\operatorname{TV}(g,[a ; b])$ is finite, moreover, both functions $f$ and $g$ are bounded (since 
they are regulated), hence the integral $\int_{a}^{b} f \mathrm{~d} g$ exists. Thus we may and will assume that $\eta_{N}>0$ and $\theta_{N}>0$ for all $N=0,1,2, \ldots$.

Choose $N=1,2, \ldots$. By the assumption that $f$ and $g$ have no common points of discontinuity, for sufficiently $\operatorname{small} \operatorname{mesh}(\pi)$, for $i=1,2, \ldots, l$ we have

$$
\sup _{a_{i-1} \leq s \leq a_{i}}\left|f(s)-f\left(a_{i-1}\right)\right| \leq \eta_{N-1}
$$

or

$$
\sup _{a_{i-1} \leq s \leq a_{i}}\left|g\left(a_{i}\right)-g(s)\right| \leq \theta_{N-1}
$$

To see this, assume that for every $h>0$, there exist $\left[a_{h} ; b_{h}\right] \subset[a ; b]$ such that $b_{h}-a_{h} \leq h$ and $\sup _{x, y \in\left[a_{h} ; b_{h}\right]}|f(y)-f(y)|>\eta_{N-1}$ and $\sup _{x, y \in\left[a_{h} ; b_{h}\right]}|g(x)-g(y)|>\theta_{N-1}$. We choose a convergent subsequence of the sequence $\left(a_{1 / n}+b_{1 / n}\right) / 2, n=1,2, \ldots$, and we see that the limit of this sequence is a point of discontinuity for both $f$ and $g$, which is a contradiction with the assumption that $f$ and $g$ have no common points of discontinuity.

Let $I$ be the set of all indices $i=1,2, \ldots, l$ for which (12) holds. Now, for $i \in I$, set $\delta_{j-1}:=$ $\eta_{N+j-1}, \varepsilon_{j}:=\theta_{N+j}, j=0,1,2, \ldots$, and define

$$
S_{i}:=\sum_{j=0}^{+\infty} 2^{j} \eta_{j-1} \cdot \operatorname{TV}^{\theta_{j}}\left(g,\left[a_{i-1} ; a_{i}\right]\right)+\sum_{j=0}^{+\infty} 2^{j} \theta_{j} \cdot \operatorname{TV}^{\eta_{j}}\left(f,\left[a_{i-1} ; a_{i}\right]\right) .
$$

By Lemma 1 we estimate

$$
\begin{aligned}
& \left|\sum_{k: s_{k-1}, s_{k} \in\left[a_{i-1} ; a_{i}\right]} f\left(s_{k-1}\right)\left[g\left(s_{k}\right)-g\left(s_{k-1}\right)\right]-f\left(a_{i-1}\right)\left[g\left(a_{i}\right)-g\left(a_{i-1}\right)\right]\right| \\
& \leq \sum_{j=0}^{+\infty} 2^{j} \delta_{j-1} \cdot \operatorname{TV}^{\varepsilon j}\left(g,\left[a_{i-1} ; a_{i}\right]\right)+\sum_{j=0}^{+\infty} 2^{j} \varepsilon_{j} \cdot \operatorname{TV}^{\delta_{j}}\left(f,\left[a_{i-1} ; a_{i}\right]\right) \\
& \quad \leq \sum_{j=0}^{+\infty} 2^{j} \eta_{N+j-1} \cdot \mathrm{TV}^{\theta_{N+j}}\left(g,\left[a_{i-1} ; a_{i}\right]\right)+\sum_{j=0}^{+\infty} 2^{j} \theta_{N+j} \cdot \operatorname{TV}^{\eta_{N+j}}\left(f,\left[a_{i-1} ; a_{i}\right]\right) \\
& \leq 2^{-N} S_{i} .
\end{aligned}
$$

Similarly,

$$
\left|f\left(v_{i}\right)\left[g\left(a_{i}\right)-g\left(a_{i-1}\right)\right]-f\left(a_{i-1}\right)\left[g\left(a_{i}\right)-g\left(a_{i-1}\right)\right]\right| \leq 2^{-N} S_{i}
$$

Hence

$$
\left|f\left(v_{i}\right)\left[g\left(a_{i}\right)-g\left(a_{i-1}\right)\right]-\sum_{k: s_{k-1}, s_{k} \in\left[a_{i-1} ; a_{i}\right]} f\left(s_{k-1}\right)\left[g\left(s_{k}\right)-g\left(s_{k-1}\right)\right]\right| \leq 2^{1-N} S_{i} .
$$

The truncated variation is a superadditive functional of the interval, from which we have

$$
\sum_{i \in I} \operatorname{TV}^{\theta_{j}}\left(g,\left[a_{i-1} ; a_{i}\right]\right) \leq \operatorname{TV}^{\theta_{j}}(g,[a ; b]), \quad \sum_{i \in I} \operatorname{TV}^{\eta_{j}}\left(f,\left[a_{i-1} ; a_{i}\right]\right) \leq \operatorname{TV}^{\eta_{j}}(f,[a ; b]) .
$$


By (14) and the last two inequalities, summing over $i \in I$ we get the estimate

$$
\begin{aligned}
& \left|\sum_{i \in I}\left\{f\left(v_{i}\right)\left[g\left(a_{i}\right)-g\left(a_{i-1}\right)\right]-\sum_{k: s_{k-1}, s_{k} \in\left[a_{i-1} ; a_{i}\right]} f\left(s_{k-1}\right)\left[g\left(s_{k}\right)-g\left(s_{k-1}\right)\right]\right\}\right| \\
& \quad \leq 2^{1-N} \sum_{i \in I} S_{i} \leq 2^{1-N} S .
\end{aligned}
$$

Now, let $J$ be the set of all indices, for which (13) holds. For $i=1,2, \ldots, l$ define

$$
T_{i}:=\sum_{j=0}^{+\infty} 2^{j} \theta_{j} \cdot \operatorname{TV}^{\eta_{j}}\left(f,\left[a_{i-1} ; a\right]\right)+\sum_{j=0}^{+\infty} 2^{j} \eta_{j} \cdot \operatorname{TV}^{\theta_{j+1}}\left(g,\left[a_{i-1} ; a_{i}\right]\right)
$$

For $i \in J$, by the summation by parts and then by Lemma 1 we get

$$
\begin{aligned}
& \left|f\left(a_{i}\right)\left[g\left(a_{i}\right)-g\left(a_{i-1}\right)\right]-\sum_{k: s_{k-1}, s_{k} \in\left[a_{i-1} ; a_{i}\right]} f\left(s_{k-1}\right)\left[g\left(s_{k}\right)-g\left(s_{k-1}\right)\right]\right| \\
& \quad=\left|\sum_{k: s_{k-1}, s_{k} \in\left[a_{i-1} ; a_{i}\right]} g\left(s_{k}\right)\left[f\left(s_{k}\right)-f\left(s_{k-1}\right)\right]-g\left(a_{i-1}\right)\left[f\left(a_{i}\right)-f\left(a_{i-1}\right)\right]\right| \\
& \quad \leq \sum_{j=0}^{+\infty} 2^{j} \theta_{N+j-1} \cdot \mathrm{TV}^{\eta_{N+j}}\left(f,\left[a_{i-1} ; a_{i}\right]\right)+\sum_{j=0}^{+\infty} 2^{j} \eta_{N+j} \cdot \mathrm{TV}^{\theta_{N+j}}\left(g,\left[a_{i-1} ; a_{i}\right]\right) \\
& \quad \leq 2^{1-N} T_{i} \leq 2^{1-N} S_{i} .
\end{aligned}
$$

Similarly, by Lemma 1,

$$
\begin{aligned}
& \left|f\left(a_{i}\right)\left[g\left(a_{i}\right)-g\left(a_{i-1}\right)\right]-f\left(v_{i}\right)\left[g\left(a_{i}\right)-g\left(a_{i-1}\right)\right]\right| \\
& \quad=\left|g\left(a_{i-1}\right)\left[f\left(v_{i}\right)-f\left(a_{i-1}\right)\right]+g\left(a_{i}\right)\left[f\left(a_{i}\right)-f\left(v_{i}\right)\right]-g\left(a_{i-1}\right)\left[f\left(a_{i}\right)-f\left(a_{i-1}\right)\right]\right| \\
& \quad \leq 2^{1-N} S_{i} .
\end{aligned}
$$

From the last two inequalities we get

$$
\begin{aligned}
\mid f\left(v_{i}\right) & {\left[g\left(a_{i}\right)-g\left(a_{i-1}\right)\right]-\sum_{k: s_{k-1}, s_{k} \in\left[a_{i-1} ; a_{i}\right]} f\left(s_{k-1}\right)\left[g\left(s_{k}\right)-g\left(s_{k-1}\right)\right] \mid } \\
\leq & \left|f\left(a_{i}\right)\left[g\left(a_{i}\right)-g\left(a_{i-1}\right)\right]-f\left(v_{i}\right)\left[g\left(a_{i}\right)-g\left(a_{i-1}\right)\right]\right| \\
& +\left|f\left(a_{i}\right)\left[g\left(a_{i}\right)-g\left(a_{i-1}\right)\right]-\sum_{k: s_{k-1}, s_{k} \in\left[a_{i-1} ; a_{i}\right]} f\left(s_{k-1}\right)\left[g\left(s_{k}\right)-g\left(s_{k-1}\right)\right]\right| \\
\leq & 2^{2-N} S_{i} .
\end{aligned}
$$

Summing over $i \in J$ and using the superadditivity of the truncated variation as anction of the interval, we get the estimate

$$
\begin{aligned}
& \left|\sum_{i \in J}\left\{f\left(v_{i}\right)\left[g\left(a_{i}\right)-g\left(a_{i-1}\right)\right]-\sum_{k: s_{k-1}, s_{k} \in\left[a_{i-1} ; a_{i}\right]} f\left(s_{k-1}\right)\left[g\left(s_{k}\right)-g\left(s_{k-1}\right)\right]\right\}\right| \\
& \quad \leq 2^{2-N} \sum_{i \in J} S_{i} \leq 2^{2-N} S .
\end{aligned}
$$


Finally, from (15) and (16) we get

$$
\left|f\left(v_{i}\right)[g(b)-g(a)]-\sum_{k=1}^{n} f\left(s_{k-1}\right)\left[g\left(s_{k}\right)-g\left(s_{k-1}\right)\right]\right| \leq 6 \cdot 2^{-N} S .
$$

A similar estimate holds for

$$
\left|\sum_{i=j}^{m} f\left(\xi_{j}\right)\left[g\left(b_{j}\right)-g\left(b_{j-1}\right)\right]-\sum_{k=1}^{n} f\left(s_{k-1}\right)\left[g\left(s_{k}\right)-g\left(s_{k-1}\right)\right]\right|,
$$

provided that the $\operatorname{mesh}(\rho)$ is sufficiently small. Hence

$$
\left|\sum_{i=1}^{l} f\left(v_{i}\right)\left[g\left(a_{i}\right)-g\left(a_{i-1}\right)\right]-\sum_{i=j}^{m} f\left(\xi_{j}\right)\left[g\left(b_{j}\right)-g\left(b_{j-1}\right)\right]\right| \leq 12 \cdot 2^{-N} S,
$$

provided that the meshes $(\pi)$ and $(\rho)$ are sufficiently small. Since $N$ may be arbitrarily large, we get the convergence of the approximating sums to an universal limit, which is the Riemann-Stieltjes integral. The estimate (4) follows directly from the proved convergence of approximating sums to the Riemann-Stieltjes integral and Lemma 1.

Using Remark 2 and reasoning similarly to the proof of Theorem 1 , we get the symmetric result.

Theorem 2 Let $f, g:[a ; b] \rightarrow \mathbb{R}$ be two regulated functions which have no common points of discontinuity. Let $\eta_{0} \geq \eta_{1} \geq \cdots$ and $\theta_{0} \geq \theta_{1} \geq \cdots$ be two sequences of non-negative numbers, such that $\eta_{k} \downarrow 0, \theta_{k} \downarrow 0$ as $k \rightarrow+\infty$. Define $\theta_{-1}:=\sup _{a \leq t \leq b}|g(b)-g(t)|$ and

$$
\tilde{S}:=\sum_{k=0}^{+\infty} 2^{k} \theta_{k-1} \cdot \operatorname{TV}^{\eta_{k}}(f,[a ; b])+\sum_{k=0}^{\infty} 2^{k} \eta_{k} \cdot \operatorname{TV}^{\theta_{k}}(g,[a ; b]) .
$$

If $\tilde{S}<+\infty$ then the Riemann-Stieltjes integral $\int_{a}^{b} f \mathrm{~d} g$ exists and one has the following estimate:

$$
\left|\int_{a}^{b} f \mathrm{~d} g-f(a)[g(b)-g(a)]\right| \leq \tilde{S}
$$

From Theorem 1, Theorem 2 and Remark 3 we also have the following.

Corollary 1 Let $f, g:[a ; b] \rightarrow \mathbb{R}$ be two regulated functions which have no common points of discontinuity, $\xi \in[a ; b]$ and $S$ and $\tilde{S}$ be as in Theorem 1 and Theorem 2, respectively. If $S<+\infty$ or $\tilde{S}<+\infty$ then the Riemann-Stieltjes integral $\int_{a}^{b} f \mathrm{~d} g$ exists and one has the following estimate:

$$
\left|\int_{a}^{b} f \mathrm{~d} g-f(\xi)[g(b)-g(a)]\right| \leq 2 \min \{S, \tilde{S}\} .
$$




\subsection{Young's theorem and the Loéve-Young inequality}

Let, for $p>0, \mathcal{V}^{p}([a ; b])$ denote the family of functions $f:[a ; b] \rightarrow \mathbb{R}$ with finite $p$-variation. Note that if $f \in \mathcal{V}^{p}([a ; b])$ then $f$ is regulated. The additional relation we will use is the following one: if $f \in \mathcal{V}^{p}([a ; b])$ for some $p \geq 1$, then for every $\delta>0$,

$$
\operatorname{TV}^{\delta}(f,[a ; b]) \leq V^{p}(f,[a ; b]) \delta^{1-p} .
$$

As far as we know, the first result of this kind, namely, $\operatorname{TV}^{\delta}(f,[a ; b]) \leq C_{f} \delta^{1-p}$ for a continuous function $f \in \mathcal{V}^{p}([a ; b])$ and some constant $C_{f}<+\infty$ depending on $f$, was proven in [11], Section 6. In [11], $\operatorname{TV}^{\varepsilon}(f,[a ; b])$ is called $\varepsilon$-variation and is denoted by $V_{f}(\varepsilon)$. However, being equipped with equation (2) we see that equation (18) follows immediately from the inequality: for any $a \leq s<t \leq b$,

$$
\max \{|f(t)-f(s)|-\delta, 0\} \leq \frac{|f(t)-f(s)|^{p}}{\delta^{p-1}}
$$

which is an obvious consequence of the estimate:

$$
\delta^{p-1} \max \{|x|-\delta, 0\} \leq\left\{\begin{array}{ll}
0 & \text { if } \delta \geq|x|, \\
|x|^{p-1} \max \{|x|-\delta, 0\} & \text { if } 0<\delta<|x|
\end{array} \leq|x|^{p}\right.
$$

for any $\delta>0$ and any real $x$.

Let us denote

$$
\|f\|_{p-\operatorname{var},[a ; b]}:=\left(V^{p}(f,[a ; b])\right)^{1 / p}
$$

and recall that $\|f\|_{\text {osc, }[a ; b]}=\sup _{a \leq s<t \leq b}|f(t)-f(s)|$. Now we are ready to state a corollary stemming from Theorem 1, which was one of the main results of [1]. The second part of this corollary is an improved version of the Loéve-Young inequality.

Corollary 2 Let $f, g:[a ; b] \rightarrow \mathbb{R}$ be two functions with no common points of discontinuity. If $f \in \mathcal{V}^{p}([a ; b])$ and $g \in \mathcal{V}^{q}([a ; b])$, where $p>1, q>1, p^{-1}+q^{-1}>1$, then the RiemannStieltjes $\int_{a}^{b} f \mathrm{~d} g$ exists. Moreover, there exists a constant $C_{p, q}$, depending on $p$ and $q$ only, such that

$$
\left|\int_{a}^{b} f \mathrm{~d} g-f(a)[g(b)-g(a)]\right| \leq C_{p, q}\|f\|_{p-\operatorname{var},[a ; b]}^{p-p / q}\|f\|_{\text {osc, }[a ; b]}^{1+p / q-p}\|g\|_{q-\operatorname{var},[a ; b]} .
$$

Proof By Theorem 1 it is enough to prove that for some positive sequences $\eta_{0} \geq \eta_{1} \geq \cdots$ and $\theta_{0} \geq \theta_{1} \geq \cdots$, such that $\eta_{k} \downarrow 0, \theta_{k} \downarrow 0$ as $k \rightarrow+\infty$ and $\eta_{-1}=\sup _{a \leq t \leq b}|f(t)-f(a)|$ one has

$$
\begin{aligned}
S & :=\sum_{k=0}^{+\infty} 2^{k} \eta_{k-1} \cdot \operatorname{TV}^{\theta_{k}}(g,[a ; b])+\sum_{k=0}^{+\infty} 2^{k} \theta_{k} \cdot \operatorname{TV}^{\eta_{k}}(f,[a ; b]) \\
& \leq C_{p, q}\|f\|_{p-\operatorname{var},[a ; b]}^{p-p / q}\|f\|_{\text {osc },[a ; b]}^{1+p / q-p}\|g\|_{q-\operatorname{var},[a ; b]} .
\end{aligned}
$$


The proof will follow from the proper choice of $\left(\eta_{k}\right)$ and $\left(\theta_{k}\right)$. Since $p^{-1}+q^{-1}>1$, we have $(q-1)(p-1)<1$. We choose

$$
\alpha \in(\sqrt{(q-1)(p-1)} ; 1), \quad \beta=\sup _{a \leq t \leq b}|f(t)-f(a)|, \quad \gamma>0,
$$

and, for $k=0,1, \ldots$, define

$$
\begin{aligned}
& \eta_{k-1}=\beta \cdot 2^{-\left(\alpha^{2} /[(q-1)(p-1)]\right)^{k}+1}, \\
& \theta_{k}=\gamma \cdot 2^{-\left(\alpha^{2} /[(q-1)(p-1)]\right)^{k} \alpha /(q-1)} .
\end{aligned}
$$

By (18) we estimate

$$
\begin{aligned}
\eta_{k-1} \cdot \mathrm{TV}^{\theta_{k}}(g,[a ; b]) \leq & \beta \cdot 2^{-\left(\alpha^{2} /[(q-1)(p-1)]\right)^{k}+1} \\
& \times V^{q}(g,[a ; b])\left(\gamma \cdot 2^{-\left(\alpha^{2} /[(q-1)(p-1)]\right)^{k} \alpha /(q-1)}\right)^{1-q} \\
= & 2^{-(1-\alpha)\left(\alpha^{2} /[(q-1)(p-1)]\right)^{k}+1} V^{q}(g,[a ; b]) \beta \gamma^{1-q},
\end{aligned}
$$

and similarly

$$
\begin{aligned}
\theta_{k} \cdot \operatorname{TV}^{\eta_{k}}(f,[a ; b]) \leq & \gamma \cdot 2^{-\left(\alpha^{2} /[(q-1)(p-1)]\right)^{k} \alpha /(q-1)} \\
& \times V^{p}(f,[a ; b])\left(\beta \cdot 2^{-\left(\alpha^{2} /[(q-1)(p-1)]\right)^{k+1}+1}\right)^{1-p} \\
= & 2^{-(1-\alpha)\left(\alpha^{2} /[(q-1)(p-1)]\right)^{k} \alpha /(q-1)+1-p} V^{p}(f,[a ; b]) \beta^{1-p} \gamma .
\end{aligned}
$$

Hence

$$
\begin{aligned}
S= & \sum_{k=0}^{+\infty} 2^{k} \eta_{k-1} \cdot \operatorname{TV}^{\theta_{k}}(g,[a ; b])+\sum_{k=0}^{+\infty} 2^{k} \theta_{k} \cdot \operatorname{TV}^{\eta_{k}}(f,[a ; b]) \\
\leq & \left(\sum_{k=0}^{+\infty} 2^{k} 2^{-(1-\alpha)\left(\alpha^{2} /[(q-1)(p-1)]\right)^{k}+1}\right) V^{q}(g,[a ; b]) \beta \gamma^{1-q} \\
& +\left(\sum_{k=0}^{+\infty} 2^{k} 2^{-(1-\alpha)\left(\alpha^{2} /[(q-1)(p-1)]\right)^{k} \alpha /(q-1)+1-p}\right) V^{p}(f,[a ; b]) \beta^{1-p} \gamma .
\end{aligned}
$$

Since $\alpha<1$ and $\alpha^{2} /[(q-1)(p-1)]>1$, we easily infer that $S<+\infty$, from which we see that the integral $\int_{a}^{b} f \mathrm{~d} g$ exists.

Moreover, denoting

$$
C_{p, q}=\max \left\{\sum_{k=0}^{+\infty} 2^{k+2-(1-\alpha)\left(\alpha^{2} /[(q-1)(p-1)]\right)^{k}}, \sum_{k=0}^{+\infty} 2^{k+2-(1-\alpha)\left(\alpha^{2} /[(q-1)(p-1)]\right)^{k} \alpha /(q-1)-p}\right\}
$$

we get

$$
S \leq \frac{1}{2} C_{p, q}\left(V^{q}(g,[a ; b]) \beta \gamma^{1-q}+V^{p}(f,[a ; b]) \beta^{1-p} \gamma\right) .
$$


Setting in this expression $\gamma=\left(V^{q}(g,[a ; b]) / V^{p}(f,[a ; b])\right)^{1 / q} \beta^{p / q}$ we obtain

$$
\begin{aligned}
S & \leq C_{p, q}\left(V^{q}(g,[a ; b])\right)^{1 / q}\left(V^{p}(f,[a ; b])\right)^{1-1 / q} \beta^{1+p / q-p} \\
& \leq C_{p, q}\|g\|_{q-\operatorname{var},[a ; b]}\|f\|_{p-\operatorname{var},[a ; b]}^{p-p / q}\|f\|_{\text {osc },[a ; b]}^{1+p / q-p} .
\end{aligned}
$$

Remark 4 Let $f, g, p, q$ and $C_{p, q}$ be the same as in Corollary 2. Using Theorem 2 instead of Theorem 1, we get the following, similar estimate:

$$
\left|\int_{a}^{b} f \mathrm{~d} g-f(a)[g(b)-g(a)]\right| \leq C_{p, q}\|f\|_{p-\mathrm{var},[a ; b]}\|g\|_{q-\mathrm{var},[a ; b]}^{q-q / p}\|g\|_{\mathrm{osc},[a ; b]}^{1+q / p-q} .
$$

From Corollary 1 and the obtained estimates, we also have for any $\xi \in[a ; b]$

$$
\begin{aligned}
& \left|\int_{a}^{b} f \mathrm{~d} g-f(\xi)[g(b)-g(a)]\right| \\
& \quad \leq 2 C_{p, q}\|f\|_{p-\mathrm{var},[a ; b]}\|g\|_{q-\mathrm{var},[a ; b]} \min \left\{\frac{\|f\|_{\mathrm{osc},[a ; b]}^{1+p / q-p}}{\|f\|_{p-\mathrm{var},[a ; b]}^{1+p / q-p}}, \frac{\|g\|_{\mathrm{osc},[a ; b]}^{1+q / p-q}}{\|g\|_{q-\mathrm{var},[a ; b]}^{1+q / p-q}}\right\} .
\end{aligned}
$$

Remark 5 From Corollary 2, reasoning in a similar way to [7], p.456, we get the following important estimate of the $q$-variation of the function $t \mapsto \int_{a}^{t} f \mathrm{~d} g$ :

$$
\begin{aligned}
\left\|\int_{a} f \mathrm{~d} g\right\|_{q-\mathrm{var},[a ; b]} & \leq\left(C_{p, q}\|f\|_{p-\mathrm{var},[a ; b]}^{p-p / q}\|f\|_{\mathrm{osc},[a ; b]}^{1+p / q-p}+\|f\|_{\infty,[a ; b]}\right)\|g\|_{q-\mathrm{var},[a ; b]} \\
& \leq\left(C_{p, q}\|f\|_{p-\mathrm{var},[a ; b]}+\|f\|_{\infty,[a ; b]}\right)\|g\|_{q-\mathrm{var},[a ; b]},
\end{aligned}
$$

where $f, g, p, q$ and $C_{p, q}$ are the same as in Corollary 2.

\section{Integral equations driven by moderately irregular signals}

Let $p \in(1 ; 2)$. The preceding section provides us with tools to solve integral equations of the following form:

$$
y(t)=y_{0}+\int_{a}^{t} F(y(s)) \mathrm{d} x(s)
$$

where $x$ is a continuous function from the space $\mathcal{V}^{p}([a ; b])$ and $F: \mathbb{R} \rightarrow \mathbb{R}$ is $\alpha$-Lipschitz. The functional $\|\cdot\|_{\mathrm{var}, p,[a ; b]}: \mathcal{V}^{p}([a ; b]) \rightarrow[0 ;+\infty)$ defined as

$$
\|f\|_{\mathrm{var}, p,[a ; b]}:=|f(a)|+\|f\|_{p-\mathrm{var},[a ; b]}
$$

is a norm and the space $\mathcal{V}^{p}([a ; b])$ equipped with this norm is a Banach space. For our purposes it will be enough to work with the following definition of a locally or globally $\alpha$ Lipschitz function when $\alpha \in(0 ; 1]$. For $x=\left(x_{1}, \ldots, x_{n}\right) \in \mathbb{R}^{n}$ we denote $\|x\|=\max _{i=1, \ldots, n}\left|x_{i}\right|$.

Definition 1 Let $F: \mathbb{R}^{n} \rightarrow \mathbb{R}$ and $\alpha \in(0 ; 1]$. For any $R>0$ we define its local $\alpha$-Lipschitz parameter $K_{F}^{\alpha}(R)$ as

$$
K_{F}^{(\alpha)}(R):=\sup \left\{\frac{|F(y)-F(x)|}{\|y-x\|^{\alpha}}: x, y \in \mathbb{R}^{n}, x \neq y,\|x\| \leq R,\|y\| \leq R\right\}
$$


and its global $\alpha$-Lipschitz parameter $K_{F}^{(\alpha)}$ as $K_{F}^{(\alpha)}:=\lim _{R \rightarrow+\infty} K_{F}(R)<+\infty$. The function $F$ will be called locally $\alpha$-Lipschitz if for every $R>0, K_{F}^{(\alpha)}(R)<+\infty$ and it will be called globally $\alpha$-Lipschitz if $K_{F}^{(\alpha)}<+\infty$.

In the case when there is no ambiguity on what is the value of the parameter $\alpha$ and what is the function $F$, we will write $K_{F}(R), K_{F}, K(R)$ or even $K$.

First we will consider the case $p-1<\alpha<1$. In this case we have the existence but no uniqueness result. We will obtain a stronger result than similar results [7], Lemma, p.459, or [8], Theorem 1.20. Namely, we will prove that there exists a solution to (20) which is an element of the space $\mathcal{V}^{p}([a ; b])$, not only an element of the space $\mathcal{V}^{q}([a ; b])$ for arbitrary chosen $q>p$. This will be possible with the use of Remark 5 .

Proposition 1 Let $p \in(1 ; 2), y_{0} \in \mathbb{R}, x$ be a continuous function from the space $\mathcal{V}^{p}([a ; b])$ and $F: \mathbb{R} \rightarrow \mathbb{R}$ be globally $\alpha$-Lipschitz where $p-1<\alpha<1$. Equation (20) admits a solution $y$, which is an element of $\mathcal{V}^{p}([a ; b])$. Moreover, $\|y\|_{\mathrm{var}, p,[a ; b]} \leq R$, where $R>0$ satisfies the equality

$$
R=\left(C_{p / \alpha, p}+2\right) K_{F}^{(\alpha)}\|x\|_{p-\operatorname{var},[a ; b]} R^{\alpha}+\left|y_{0}\right|+|F(0)|\|x\|_{p-\mathrm{var},[a ; b]},
$$

with $C_{p / \alpha, p}$ being the same as in Corollary 2 and Remark 5.

Now we proceed to the proof of Proposition 1. We will proceed in a standard way, but with the more accurate estimate of Remark 5 we will be able to obtain the finiteness of $\|\cdot\|_{\mathrm{var}, p,[a ; b]}$ norm of the solution.

Proof Let $f \in \mathcal{V}^{p}([a ; b])$. By [8], Lemma 1.18, $F(f(\cdot)) \in \mathcal{V}^{p / \alpha}([a ; b])$ and since $\alpha / p+1 / p>1$, we may apply Remark 5 and define the operator $T: \mathcal{V}^{p}([a ; b]) \rightarrow \mathcal{V}^{p}([a ; b])$,

$$
T f:=y_{0}+\int_{a} F(f(t)) \mathrm{d} x(t)
$$

Denote $K=K_{F}^{(\alpha)}$. Using Remark 5 and [8], Lemma 1.18, we estimate

$$
\begin{aligned}
\|T f\|_{\mathrm{var}, p,[a ; b]}= & \left\|y_{0}+\int_{a} F(f(t)) \mathrm{d} x(t)\right\|_{\operatorname{var}, p,[a ; b]} \\
\leq & \left\|\int_{a}[F(f(t))-F(f(a))] \mathrm{d} x(t)\right\|_{p-\operatorname{var},[a ; b]}+|F(f(a))|\|x\|_{p-\operatorname{var},[a ; b]}+\left|y_{0}\right| \\
\leq & \left(C_{p / \alpha, p}\|F(f(\cdot))\|_{p / \alpha-\operatorname{var},[a ; b]}+\|F(f(\cdot))-F(f(a))\|_{\infty,[a ; b]}+|F(f(a))|\right) \\
& \times\|x\|_{p-\operatorname{var},[a ; b]}+\left|y_{0}\right| \\
\leq & \left(\left(C_{p / \alpha, p}+1\right)\|F(f(\cdot))\|_{p / \alpha-\operatorname{var},[a ; b]}+|F(f(a))|\right)\|x\|_{p-\operatorname{var},[a ; b]}+\left|y_{0}\right| \\
\leq & \left(\left(C_{p / \alpha, p}+1\right) K\|f\|_{p-\operatorname{var},[a ; b]}^{\alpha}+|F(f(a))|\right)\|x\|_{p-\operatorname{var},[a ; b]}+\left|y_{0}\right| .
\end{aligned}
$$

By the Lipschitz property,

$$
|F(f(a))| \leq K|f(a)|^{\alpha}+|F(0)| \leq K\|f\|_{\mathrm{var}, p,[a ; b]}^{\alpha}+|F(0)| .
$$


Denoting

$$
A=\left(C_{p / \alpha, p}+2\right) K\|x\|_{p-\operatorname{var},[a ; b]} \quad \text { and } \quad B=\left|y_{0}\right|+|F(0)|\|x\|_{p-\operatorname{var},[a ; b]},
$$

from (21) we get

$$
\|T f\|_{\mathrm{var}, p,[a ; b]} \leq A\|f\|_{\mathrm{var}, p,[a ; b]}^{\alpha}+B .
$$

For $\alpha<1$ let $R$ be the least positive solution of the inequality $R \geq A \cdot R^{\alpha}+B$ (i.e. $R=$ $\left.A \cdot R^{\alpha}+B\right)$. From (23) we see that the operator $T$ maps the closed ball $\mathcal{B}(R)=\left\{f \in \mathcal{V}^{p}([a ; b])\right.$ : $\left.\|f\|_{\mathrm{var}, p,[a ; b]} \leq R\right\}$ to itself.

Now, for $f, g \in \mathcal{V}^{p}([a ; b])$ we are going to investigate the difference $T f-T g$. Again, using Remark 5 and the Lipschitz property we estimate

$$
\begin{aligned}
\| T f- & T g \|_{\mathrm{var}, p,[a ; b]} \\
= & \left\|\int_{a}[F(f(t))-F(g(t))] \mathrm{d} x(t)\right\|_{p-\mathrm{var},[a ; b]} \\
\leq & \left\|\int_{a}[F(f(t))-F(g(t))-\{F(f(a))-F(g(a))\}] \mathrm{d} x(t)\right\|_{p-\mathrm{var},[a ; b]} \\
& +|F(f(a))-F(g(a))|\|x\|_{p-\operatorname{var},[a ; b]} \\
\leq & \left(C_{p / \alpha, p}+1\right)\|F(f(\cdot))-F(g(\cdot))\|_{p / \alpha-\operatorname{var},[a ; b]}^{(p-1) / \alpha}\|F(f(\cdot))-F(g(\cdot))\|_{\mathrm{osc},[a ; b]}^{(\alpha+1-p) / \alpha} \\
& \times\|x\|_{p-\mathrm{var},[a ; b]}+|F(f(a))-F(g(a))|\|x\|_{p-\mathrm{var},[a ; b]} \\
\leq & \left(C_{p / \alpha, p}+1\right) K\left(\|f\|_{p-\operatorname{var},[a ; b]}^{\alpha}+\|g\|_{p-\mathrm{var},[a ; b]}^{\alpha}\right)^{(p-1) / \alpha}\|f-g\|_{\mathrm{osc},[a ; b]}^{\alpha+1-p} \\
& \times\|x\|_{p-\mathrm{var},[a ; b]}+K|f(a)-g(a)|^{\alpha}\|x\|_{p-\mathrm{var},[a ; b] .}
\end{aligned}
$$

From (24) we see that $T$ is continuous. Moreover, from the first inequality in Remark 4 and the continuity of $x$ we see that functions belonging to the image $T(\mathcal{B}(R))$ are equicontinuous. Let $\mathcal{U}$ be the closure of the convex hull of $T(\mathcal{B}(R)$ ) (in the topology induced by the norm $\left.\|\cdot\|_{\mathrm{var}, p,[a ; b]}\right)$. It is easy to see that functions belonging to $\mathcal{U}$ are also equicontinuous. Moreover, $\mathcal{U} \subset \mathcal{B}(R)$ (since $T(\mathcal{B}(R)) \subset \mathcal{B}(R)$ and $\mathcal{B}(R)$ is convex) and $T(\mathcal{U}) \subset \mathcal{U}$ (since $T(\mathcal{U}) \subset T(\mathcal{B}(R)))$. Now, let $\mathcal{V}=T(\mathcal{U})$. From the equicontinuity of $\mathcal{U}$, the Arzela-Ascoli theorem and (24) we see that the set $\mathcal{V}$ is compact in the topology induced by the norm $\|\cdot\|_{\mathrm{var}, p,[a ; b]}$. Thus, by the fixed-point theorem of Schauder, we see that there exists a point $y \in \mathcal{U}$ such that $T y=y$.

Now we will consider the case $\alpha=1$.

Fact 1 Let $p \in(1 ; 2), y_{0} \in \mathbb{R}, x$ be a continuous function from the space $\mathcal{V}^{p}([a ; b])$ and $F: \mathbb{R} \rightarrow \mathbb{R}$ be globally 1-Lipschitz. Equation (20) admits a solution $y$, which is an element of $\mathcal{V}^{p}([a ; b])$.

Proof To prove the assertion we may proceed in a similar way to the proof of Proposition 1. The only thing we need is to ensure that the inequality $R \geq A \cdot R+B$, where $A$ and $B$ are 
defined in display (22), holds for sufficiently large $R$. This may be achieved by splitting the interval $[a ; b]$ into small intervals, such that $A<1$ on each of these intervals, and then solving equation (20) on each of these intervals with the initial condition being equal the terminal value of the solution on the preceding interval. This is possible since for any $\varepsilon>0$ there exists $\delta>0$ such that for any $[c ; c+\delta] \subset[a ; b],\|x\|_{p-\operatorname{var},[c ; c+\delta]} \leq \varepsilon$, which is a consequence of the fact that the function $[a ; b] \ni t \mapsto\|x\|_{p-\mathrm{var},[a ; t]}$ is continuous and we have $\|x\|_{p-\operatorname{var},[a ; c+\delta]} \geq\|x\|_{p-\operatorname{var},[a ; c]}+\|x\|_{p-\operatorname{var},[c ; c+\delta]}$.

Competing interests

The author declares to have no competing interests.

\section{Acknowledgements}

I would like to thank Raouf Ghomrasni for drawing my attention to paper [11] and Professor Terry Lyons for drawing my attention to his paper [7].

Received: 5 August 2015 Accepted: 21 November 2015 Published online: 03 December 2015

\section{References}

1. Young, LC: An inequality of the Hölder type, connected with Stieltjes integration. Acta Math. 67(1), 251-282 (1936)

2. Friz, PK, Victoir, NB: Multidimensional Stochastic Processes as Rough Paths: Theory and Applications. Cambridge Studies in Advanced Mathematics, vol. 120. Cambridge University Press, Cambridge (2010) xiv+656 pp.

3. Young, LC: General inequalities for Stieltjes integrals and the convergence of Fourier series. Math. Ann. 115, 581-612 (1938)

4. D'yačkov, AM: Conditions for the existence of Stieltjes integral of functions of bounded generalized variation. Anal. Math. 14, 295-313 (1988)

5. Dudley, RM, Norvaiša, R: Concrete Functional Calculus. Springer Monographs in Mathematics, Springer, New York (2010) 683 pp.

6. Appel, J, Banaś, J, Díaz, NJM: Bounded Variation and Around. de Gruyter, Berlin (2013)

7. Lyons, T: Differential equations driven by rough signals (I): an extension of an inequality of L. C. Young. Math. Res. Lett. 1, 451-464 (1994)

8. Lyons, TJ, Caruana, M, Lévy, T: Differential Equations Driven by Rough Paths. Lecture Notes in Mathematics, vol. 1902. Springer, Berlin (2007)

9. Łochowski, RM: On a generalisation of the Hahn-Jordan decomposition for real càdlàg functions. Colloq. Math. 132(1), 121-138 (2013)

10. Łochowski, RM, Ghomrasni, R: The play operator, the truncated variation and the generalisation of the Jordan decomposition. Math. Methods Appl. Sci. 38(3), 403-419 (2015). doi:10.1002/mma.3077

11. Tronel, G, Vladimirov, AA: On BV-type hysteresis operators. Nonlinear Anal., Theory Methods Appl. 39(1), 79-98 (2000). doi:10.1016/S0362-546X(98)00168-0

\section{Submit your manuscript to a SpringerOpen ${ }^{\ominus}$ journal and benefit from:}

- Convenient online submission

- Rigorous peer review

- Immediate publication on acceptance

- Open access: articles freely available online

- High visibility within the field

- Retaining the copyright to your article 